\title{
Varicella zoster myocarditis progressing to cardiomyopathy and cardiac transplantation
}

\author{
Andrew Tsintsof, Warwick J Delprado, Anne M Keogh
}

Cardiopulmonary

Transplant Unit, St Vincent's Hospital, Darlinghurst, Sydney, Australia A Tsintsof W J Delprado A M Keogh

Correspondence to: Dr Anne Keogh, Cardiopulmonary Transplant Unit, St Vincent's Hospital, Darlinghurst, 2010, NSW, Sydney, Australia.

\begin{abstract}
The case of a 12 year old schoolgirl with heart failure due to varicella myocarditis is reported. Heart failure and cardiogenic shock were evident 21 days after the appearance of the rash, and cardiac transplantation was performed two weeks later. Myocarditis is a serious complication of varicella zoster infection and heart failure may be fulminant. Endomyocardial biopsy changes consistent with myocarditis were documented six days after the start of heart failure. The histological changes, however, developed into those of idiopathic dilated cardiomyopathy (with anisonucleosis and fibre width variation) over a seven day period. This case provides further evidence for the link between viral myocarditis and idiopathic cardiomyopathy and underlines the value of immediate endomyocardial biopsy in heart failure of recent onset. Cardiac transplantation led to a rapid and full recovery.
\end{abstract}

(Br Heart f 1993;70:93-95)

Primary infection with varicella zoster virus (chickenpox) is characterised by a generalised pruritic vesicular rash, usually without significant systemic illness. Serious complications such as encephalitis, pneumonitis, pancreatitis, and nephritis may occur, however, particularly in neonates, immunocompromised patients, and sometimes normal adults. ${ }^{1}$ Myocarditis is an uncommon but serious complication. We report on a previously healthy child who developed biopsy-proven myocarditis after a primary infection with varicella zoster virus. This rapidly developed into a dilated cardiomyopathy (with histological changes consistent with this diagnosis) and the girl then needed cardiac transplantation.

\section{Case report}

A $51 \mathrm{~kg}, 12$ year old schoolgirl presented with a five day history of vomiting, exertional dyspnoea, cough, and extreme lethargy that started 21 days after the start of an itchy, vesiculopapular rash diagnosed as chicken pox. Initial treatment comprised salicylates and two weeks isolation. Previously the patient had experienced no major illness. There was no relevant family history of heart disease.
On presentation she was pale, peripherally cool, unwell, and lethargic. Heart rate was 145 beats/minute, blood pressure was 95/60 $\mathrm{mm} \mathrm{Hg}$, and she was afebrile. Jugular venous pressure was elevated to the jaw, the apex beat was displaced laterally, and a third heart sound was audible. Lung fields were clear and there was no hepatomegaly or peripheral oedema.

A chest radiograph showed cardiomegaly, pulmonary venous congestion, interstitial oedema, and small bilateral pleural effusions. The electrocardiogram showed sinus tachycardia, a nonspecific intraventricular conduction defect, poor anterior $\mathrm{R}$ wave progression and inferolateral ST-T wave changes. The white cell count was $12.7 \times 10^{9} / \mathrm{I}$ (normal $4-11 \times 10^{9} / \mathrm{I}$ ) with a neutrophilia (neutrophils $71 \%$, lymphocytes $14 \%$, monocytes $15 \%$ ). Serum potassium $(3.2 \mathrm{mmol} / \mathrm{l})$ and magnesium $0.74 \mathrm{mmol} / \mathrm{l}$ ) concentrations were subnormal. Serum albumin, creatinine, and urea concentrations, and hepatic transaminase activities were normal. Serological studies confirmed a recent primary varicella zoster infection with positive IgM and viral antibody titre of 32 (complement fixation test). Coxsackie, echo, influenza A and B, measles, cytomegalovirus, and Epstein Barr viral titres were all negative. Thyroid function testing was consistent with a sick euthyroid state. Echocardiography showed a considerably dilated, diffusely hypokinetic left ventricle with end diastolic diameter $73 \mathrm{~mm}$ (normal $35-56 \mathrm{~mm})$ and dilated left atrium $(47 \mathrm{~mm}$, normal $<40 \mathrm{~mm}$ ). There was moderate mitral regurgitation, mild tricuspid regurgitation, and a large apical thrombus. Left ventricular ejection fraction was $22 \%$ on radionuclide ventriculography. Endomyocardial biopsy performed 28 days after the start of the rash showed focal aggregates of mononuclear cells ( $>5$ lymphocytes/high power field), anisonucleosis, variation in fibre diameter and scattered necrotic myocytes with lymphocyte "cuffing" (fig).

A diagnosis of varicella myocarditis was made. Fluid and salt restriction, frusemide, digoxin, captopril, and anticoagulation with heparin were started. In an attempt to limit myocardial destruction, $500 \mathrm{mg} /$ day methylprednisolone was given intravenously for three consecutive days followed by a tapered dose of oral prednisolone ( $1 \mathrm{mg} / \mathrm{kg} /$ day reducing over 14 days to $0.2 \mathrm{mg} / \mathrm{kg} /$ day), and $1 \mathrm{mg} / \mathrm{kg} /$ day azathioprine orally was started. She also received acyclovir (500 mg eight hourly) intravenously. By 30 days after the 

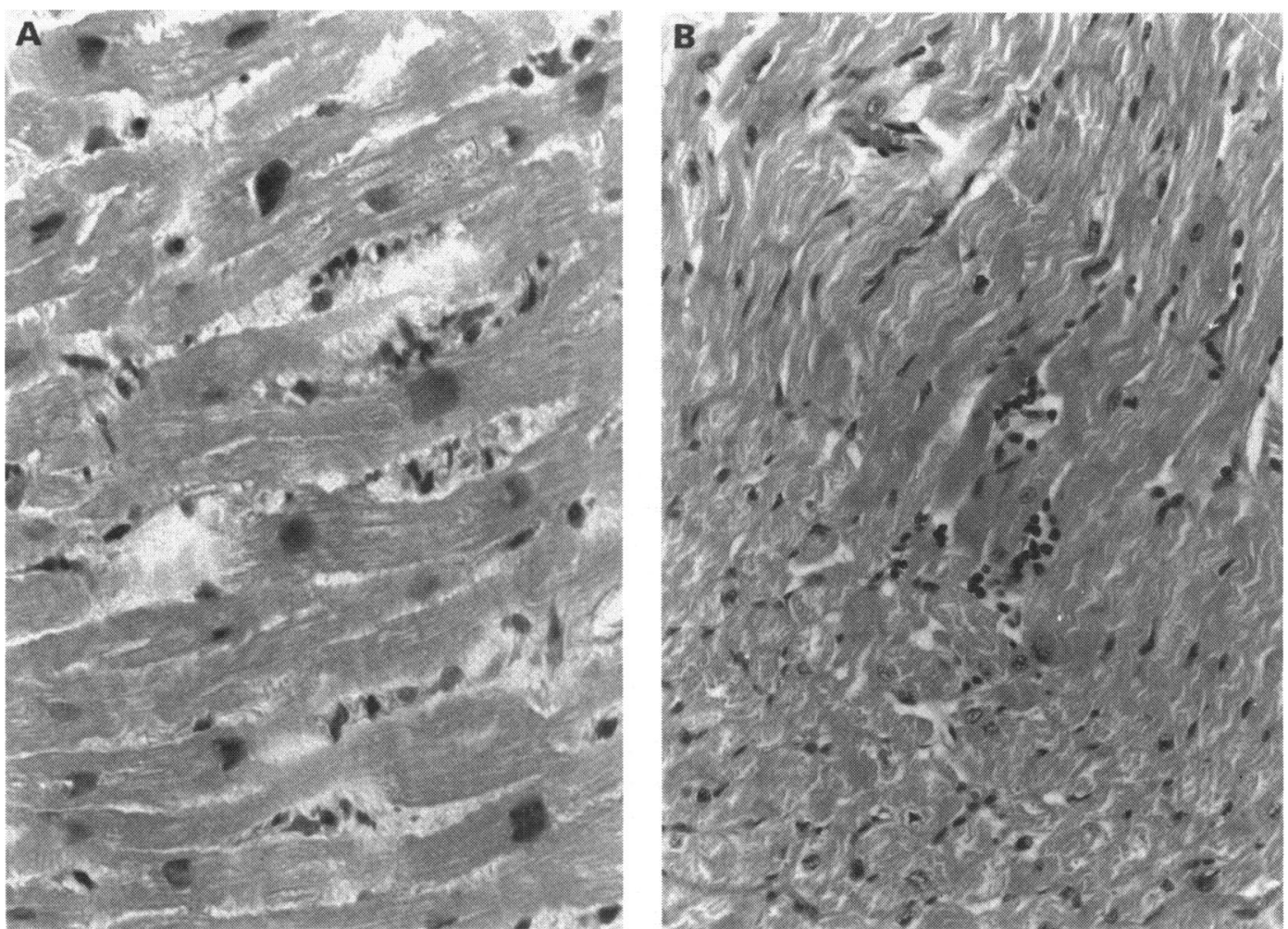

(A) Endomyocardial biopsy: necrotic, discontinuous myocyte with lymphocytes (haematoxylin and eosin stain, originally $40 \times$ magnification). (B) Endomyocardial biopsy. Central necrotic myocyte with homogeneous dense appearance to the cytoplasm. There are several related foci of dark staining lymphocytes (haematoxylin and eosin stain, originally $20 \times$ magnification).

start of the rash, intravenous dopamine (4 $\mu \mathrm{g} / \mathrm{kg} / \mathrm{min}$ ) was required for persistent sinus tachycardia and hypotension $(70 / 50 \mathrm{~mm} \mathrm{Hg})$. Left anterior pleuritic chest pain led to a ventilation perfusion scan that reported low probability of a pulmonary embolus.

Right heart catheterisation showed right atrial pressure to be $3 \mathrm{~mm} \mathrm{Hg}$, mean pulmonary artery pressure $20 \mathrm{~mm} \mathrm{Hg}$, pulmonary capillary wedge pressure $15 \mathrm{~mm} \mathrm{Hg}$, cardiac index $2 \cdot 1$ litres $/ \mathrm{min} / \mathrm{m}^{2}$ (on dopamine) and pulmonary vascular resistance 1.6 Wood units. An electrocardiogram on day 31 of the illness showed transient $2 \mathrm{~mm} \mathrm{ST}$ elevation in the anterior chest leads, resolving after four hours without $Q$ wave development. Serum creatine kinase and its myocardial subfraction were normal. Frequent multifocal venticular extrasystoles and salvoes of ventricular tachycardia occurred and there was progressive broadening of the intraventricular conduction defect on electrocardiography. It was decided to perform an urgent cardiac transplant as lack of response to treatment was obvious. On the 35th day after development of the rash, a suitable donor became available. Orthotopic transplantation was performed with an unremarkable postoperative course. The patient resumed school four months later and remains well two years after transplantation.

Examination of the explanted heart showed a very dilated, thin walled left ventricle and thickened epicardial surface anteriorly with numerous petechial haemorrhages. There were areas of fibrosis at the bases of the right ventricular papillary muscles. The epicardial arteries were normal. Histological examination showed features consistent with a dilated cardiomyopathy with anisonucleosis and fibre width variation. There was no evidence of lymphocytic or mononuclear cell infiltration, or of myocyte necrosis, and little or no fibrous tissue.

\section{Discussion}

Clinically evident chickenpox myocarditis is rare, yet in this case was severe enough to require cardiac transplantation. Vomiting was initially misdiagnosed as gastroenteritis before heart failure was correctly identified. RNA probe techniques were not available to assess the presence of virus in the myocardium, but serological evidence of a recent infection was shown.

The diagnosis of myocarditis was based on the histological changes of an inflammatory mononuclear infiltration near necrotic myocytes. ${ }^{2}$ The inflammatory infiltration was not intense and the myocyte necrosis not widespread, yet the clinical picture of severely impaired systolic function was irrefutable. This is in keeping with the well reported lack of correlation between the severity of histological changes in the myocardium and the degree of myocardial dysfunction. ${ }^{3}$ This may be partly explained by the effects of cytokines released from lymphocytes, which can be functional depressants to the myocardium.

Seven days after cardiac biopsy, the explanted heart was indistinguishable from one with idiopathic cardiomyopathy. The lack of development of fibrosis probably 
reflects the short time of illness. This rapid change in histological appearance adds to other evidence linking acute myocarditis with idiopathic dilated cardiomyopathy. ${ }^{45}$ It also shows the potentially rapid transition of myocarditis to postmyocarditic cardiomyopathy and the need to biopsy as early as possible in cases of heart failure of recent onset. Repeat endomyocardial biopsy has been advocated between three weeks and three months after the initiation of treatment for myocarditis. ${ }^{6}$ Other reports, however indicate that early repeat endomyocardial biopsy (within four days) may show remarkable changes in histopathology. ${ }^{5}$

Chickenpox affects $90 \%$ of children during the first decade of life. ${ }^{7}$ It seems possible that subclinical varicella myocarditis may underlie entities previously classified as idiopathic cardiomyopathy and such a myocardial insult could leave the heart more prone to dysfunction when stressed in later life.

Acyclovir was given after the varicelliform skin manifestations had resolved. We are aware of three reports in which acyclovir has been used in varicella myocarditis. ${ }^{8-10}$ These patients received acyclovir within five days of the start of exanthem. One patient recovered fully, another was left with heart block and left ventricular impairment, and third patient died. It seems certain that our patient would have died had a donor not become available. The use of acyclovir did not appear to be beneficial. Acyclovir may be useful against Varicella in normal adults, ${ }^{11}$ in preventing dissemination in immunocompromised children ${ }^{1213}$ and in shortening the duration of fever and skin manifestations in normal children when given orally within 24 hours of developing the rash. ${ }^{14}$ Acyclovir may thus be potentially beneficial in varicella myocarditis if presentation occurs during the stage of viral replication within the myocardium. This is more likely early in the course of the illness while new skin lesions are still appearing.

Steroid treatment and azathioprine were also given in an attempt to prevent further myocardial destruction. The place of immunosuppression, however, in the treatment of myocarditis, particularly that mediated by viruses, remains controversial. ${ }^{615-17}$ In this case, there was deterioration in clinical state despite reduction in inflammatory infiltrate.

In conclusion, the histopathological changes seen in this case are evidence that acute chickenpox myocarditis can progress rapidly to dilated cardiomyopathy. Such a transition may occur within a seven day period. Chickenpox is a common condition and it is conceivable that subclinical infection or myocarditis may contribute to some cases of idiopathic dilated cardiomyopathy.

1 Straus S, Ostrove J, Inchauspe G, et al. Varicella-zoster virus infections. Ann Intern Med 1988;108:221-37.

2 Aretz H, Billingham M, Edwards W, et al. Myocarditis: a histopathologic definition and classification. $A m f$ Cardiovasc Pathol 1986:1:3-14

3 Schwarz F, Mall G, Zebe H, et al. Determinants of survival in patients with congestive cardiomyopathy: quantitative morphological findings and left ventricular hemodynamics. Circulation 1984;70:923-8.

4 Billingham $M$, Tazelaar $H$. The morphological progression of viral myocarditis. Postgrad Med 7 1986;62:581-4.

5 Keogh A, Billingham M, Schroeder J. Rapid histological changes in endomyocardial biopsy specimens after myocarditis. Br Heart f 1990;64:406-8.

6 Hosenpud J, McAnulty J, Niles N. Lack of objective improvement in ventricular systolic function in patients with myocarditis treated with azathioprine and predwith myocarditis treated with azathioprine
nisone. $\mathrm{F} \mathrm{Am}$ Coll Cardiol 1985;6:797-801.

7 Guess H. Population-based studies of Varicella complications. Paediatrics 1986;78:723-7.

8 Coppack S, Doshi R, Ghose A. Fatal Varicella in a healthy young adult. Postgrad Med f 1985;61:529-31.

9 Ettedgui J, Ladusans E, Bamford M. Complete heart block as a complication of Varicella. Int $\mathcal{F}$ Cardiol 1987; 14:362-5.

10 Waagner D, Murphy T. Varicella myocarditis. Pediatric Infections Diseases 1990;9:360-3.

11 Al-Nakib W, Al-Kandari S, El-Khalik D, El-Shirbiny A. A randomised controlled study of intravenous acyclovir (Zovirax) against placebo in adults with chicken pox. f Infect Dis 1983;6(suppl 1):49-56.

12 Prober C, Kirk L, Keeney R. Acyclovir therapy of chickenpox in immunosuppressed children: a collaborative enpox in immunosuppressed ch.

13 Nyerges G, Meszner Z, Gyarmati E, Kerpel-Fronius S. Acyclovir prevents dissemination of Varicella in immunocompromised children. F Infect Dis 1988;157: munoco $309-13$.

14 Balfour H, Kelly J, Suarez C, et al. Acyclovir treatment of Varicella in otherwise healthy children. F Paediatr 1990; 116:633-9.

15 Hobbs R, Pelegrin D, Ratliff $\mathrm{N}$, et al. Lymphocytic myocarditis and dilated cardiomyopathy: treatment with immunosuppressive agents. Cleve Clin $\mathcal{F}$ Med 1989;56: 628-35.

16 Latham R, Mulrow J, Virmani R, Robinowitz M, Moody $\mathrm{J}$. Recently diagnosed idiopathic dilated cardiomyopathy: incidence of myocarditis and efficacy of prednisone therapy. Am Heart F 1989;117:876-82.

17 Salvi A, DiLenarda A, Dreas L, Silvestri F, Camerini F. Immunosuppressive treatment in myocarditis. Int $\mathcal{f}$ Cardiol 1989;22:329-38. 